\title{
Characteristics of near-surface electrokinetic coupling
}

\author{
David Beamish \\ British Geological Survey, Keyworth, Nottingham, NG12 5GG, UK.E-mail:dbe@bgs.ac.uk
}

Beamish, D., 1999. Characteristics of near-surface electrokinetic coupling. Geophys. J. Int., 137, 231-242.

doi: 10.1046/j.1365-246x.1999.00785.x

\section{SUMMARY}

Naturally occurring electric potentials at the Earth's surface are traditionally studied using self-potential geophysics. Recent theoretical and experimental work has reinvestigated the manner in which the measurement can be made dynamically using a pressure source. The methodology, often referred to as seismoelectric, relies on electrokinetic coupling at interfaces in the streaming potential coefficient. The ultimate aim of the developing methodologies lies in the detection of zones of high fluid mobility (permeability) and fluid geochemical contrasts within the subsurface. As yet there are no standard methods of recording and interpretation: the technique remains experimental. Field measurements are made using a seismic source and by recording electric voltage across arrays of surface dipoles. This study presents observational characteristics of electrokinetic coupling based on experiments carried out in a wide range of environments. Theory concerning the coupled elastic and electromagnetic wave equations in a saturated porous medium is discussed. It is predicted that coupling will produce electromagnetic radiation patterns from vertical electric dipoles generated at interfaces. Surface- and body-wave coupling mechanisms should provide different time-distance patterns. Vertical electric dipole radiation sources are modelled and their spatial characteristics presented. A variety of experimental configurations have been used, and geometries that exploit phase asymmetry to enhance the separation of signal and noise are emphasized. The main experimental results presented are detailed observations in the immediate vicinity of the source. Simultaneous arrivals across arrays of surface dipoles are not common. The majority of such experiments have indicated that shot- symmetric voltages which display lowvelocity moveout are the dominant received waveforms.

Key words: electrical streaming currents, electrokinetic, electromagnetic, hydraulic transport. 


\section{INTRODUCTION}

A number of recent experimental and theoretical papers have discussed the generation of electromagnetic fields when acoustic waves from a seismic source propagate through the subsurface. The methodology at the field scale is referred to as either seismoelectric (Butler et al. 1996; Russell et al. 1997), electroseismic (Mikhailov et al. 1997) or electrokinetic conversion (Thompson \& Gist 1993). Electrokinetic observations use an acoustic wave as source and record electrical voltage dynamically. Coupling may occur at the pore scale through the electrical double layer, which provides an existing natural potential (the zeta potential) in all rock/fluid systems (Bockris \& Reddy 1970). The zeta potential is described at the macroscopic level by the streaming potential coefficient. The ultimate aim of the developing methodologies lies in the detection of zones of high fluid mobility (permeability) and fluid geochemical contrasts within the subsurface.

Historically, geophysical aspects of electrokinetic conversion have appeared intermittently. In the first volume of the journal Geophysics, Thompson (1936) proposed that the coupling of seismic and electric fields could be used as an exploration tool. Ivanov (1939) made observations of electric fields generated by explosions. It was noted that there was a phase reversal in the electric field recorded when the elastic wave was generated on opposite sides of the electrode spread. The effect observed was discussed in terms of the solid and liquid phases present in the rock mass. Although Thompson (1936) and Long \& Rivers (1975) discuss a coupling mechanism in terms of resistivity modulation, the mechanism discussed here is that of electrokinetic coupling due to double-layer displacement.

Observational field studies of electrokinetic phenomena appeared in western journals in the 1950s (Martner \& Sparks 1959), the 1960s (Broding et al. 1963), the 1970s (Long \& Rivers 1975) and the 1980s (Murty 1985). Soviet experiments are described by Parkhomenko (1971). The experiments include both laboratory and borehole investigations of electro- kinetic effects (Parkhomenko \& Gaskarov 1971; Gaskarov \& Parkhomenko 1974). Thompson \& Gist (1993) published the results of a systematic study of the potential of electroseismic effects in the context of deep exploration for oil and gas. The study, both theoretical and field-based, predicted measurable electric field effects. The study concluded by suggesting the greatest potential for successful application lay in shallow exploration (for example for aquifers).

The present study largely concerns the observational characteristics of electrokinetic coupling based on experiments carried out in a wide range of hydrogeological environments. The effects that have been observed are pervasive and appear to be associated with both the unsaturated and saturated subsurface. The experiments reveal some general characteristics that can be routinely observed using (predominantly) a hammer/plate seismic source. Although there exist some general theoretical predictions concerning the likely behaviour of electrokinetic effects, detailed predictions and numerical modelling are limited. The present study addresses the general behaviour of electrokinetic observations and does not attempt detailed interpretation.

The observational studies considered here are essentially an attempt to both generate and measure the effects of dynamic streaming potentials. The background to these attempts lies in the steady-state principles of selfpotential geophysics, which are discussed first. In order to understand the behaviour of electrokinetic phenomena, the fully coupled behaviour of elastic and electromagnetic wave interactions in porous media must be examined. The basic principles of the acoustic source, together with recent theoretical predictions of the manner in which the source can generate electrokinetic coupling, are considered. From the work of Pride \& Haartsen (1996) and Haartsen et al. (1998) it will be appreciated that compressional (fast and slow) and shear waves together with reflected events have to be considered to obtain a complete description of the coupling. Only an outline description is provided here.

Theory predicts that a macroscopic, charge distribution may be generated at an interface in the streaming potential coefficient. The subsurface property dependences involved in electrokinetic coupling are not simple. The relationships suggest that all interfaces involving changes in the type of pore fluid (for example air or water), rock type and the microstructural properties (porosity and permeability) have to be considered. The electromagnetic oscillation should vary with the time signature of the acoustic wave and should have the characteristic of a vertical electric dipole. The electric and magnetic fields associated with 
subsurface vertical electric dipoles have been modelled to provide an indication of the spatial scale of typical near-surface effects and the depth sensitivity of arrays of surface dipole receivers. Field acquisition systems and geometries that exploit the phase asymmetry of the vertical electric dipole to enhance the separation of signal and noise are discussed. The main experimental results presented here are detailed observations in the immediate vicinity of the acoustic source using split-spread configurations. The nature of the received voltages as a function of electrode type and electrode length are considered. A large number of simple experiments have been carried out to assess the received voltage characteristics in a wide variety of environments. Although some effects are site-specific it is possible to summarize a number of general findings.

Ultimately, investigations into electrokinetic effects require extensive field experiments to map the behaviour of both acoustic and electromagnetic fields as a function of space and time. The results of a number of such multichannel experiments are presented. The experiments assist in the separation of predicted coupling effects due to vertically propagating acoustic waves, which should produce instantaneous effects, and horizontally propagating waves, which produce time-delayed (moveout) effects across arrays of surface dipoles.

\section{ELECTROKINETC COUPLING}

Electrokinetic phenomena are defined as arising from the relative motion between a charged surface and its associated double layer (Bockris \& Reddy 1970). If the electrokinetic/streaming potential $(\nabla V)$ represents the potential induced by a pressure gradient $(\nabla P)$ in a porous medium then, under steady-state equilibrium,

$\nabla V=C \nabla P$

where $C$ is referred to as the streaming potential coefficient (Overbeek 1952); typical values (-12 to over $350 \mathrm{mV}$ $\left.\mathrm{atm}^{-1}\right)$ in a variety of rock types are given in Ahmed (1964).

Eq. (1) is the classical Helmholtz-Schmoluchowski equation; C is independent of any microstructural (pore) parameter and is given by

$$
C=\varepsilon \xi / \eta \sigma
$$

where $\varepsilon, \xi, \eta$ and $\sigma$ are the fluid dielectric constant, the zeta potential, the fluid dynamic viscosity and the fluid electrical conductivity, respectively. A study of the dependence of the zeta potential in general rockwater systems is given by Ishido \& Mizutani (1981). Pride \& Morgan (1991) summarize the dependence of the streaming potential coefficient (the zeta potential) with electrolyte $(\mathrm{NaCl}, \mathrm{KCl})$ molarity. The magnitude of the zeta potential and hence streaming potential increases with decreasing electrolyte concentration, so that the largest streaming potentials are associated with fresh (non-saline) fluid environments.

Thompson \& Gist (1993) discuss the concept of Fresnel zones associated with a down-going seismic pulse and which are centred directly beneath the shot-point. The authors emphasize the importance of the first Fresnel zone in relation to the electrokinetic coupling mechanism at subsurface interfaces. It is the first Fresnel zone, which exhibits dipolar symmetry, that is expected to produce the largest electromagnetic field.

It is important also to note that, in the case of a compressional acoustic wave propagating through a homogenous porous material, electrokinetic coupling produces a constant electric field confined to the wave (Fitterman 1978; Pride \& Haartsen 1996). Independently propagating electromagnetic waves are not generated. When the acoustic wave traverses a boundary separating regions with different streaming potential coefficients, a charge separation that oscillates at the acoustic frequency is produced. This charge oscillation generates an electromagnetic field that can be observed at the surface. From the previous equations, the magnitude of the field will depend on the electrochemical properties of the rock/fluid and the mobility of the pore fluid.

In order to understand the behaviour of electrokinetic phenomena, the fully coupled behaviour of elastic and electro- magnetic wave interactions in porous media must be examined. The theories of quasi-static and dynamic poroelasticity for fully saturated media were presented by Biot $(1956,1962)$ and are the classical papers on the 
subject. More recent descriptions of Biot theory in relation to electrokinetic effects are given by Neev \& Yeatts (1989), Pride (1994) and Pride \& Haartsen (1996). Electrokinetic streaming current behaviour in homogenous poroelastic media is discussed by Haartsen et al. (1998), and numerical modelling of a single subsurface interface is presented by Mikhailov et al. (1997). In the latter paper, electrokinetic coupling effects are discussed according to two mechanisms at subsurface interfaces. Fig. 1 provides a schematic summary of these two mechanisms. The first occurs when a spherical $P$ wave traverses the boundary directly beneath the shot-point. This mechanism is referred to here as Fresnel-zone coupling and would provide instantaneous electromagnetic arrivals across arrays of surface dipoles. The second mechanism is the electromagnetic field generated when a refracted head wave travels along an interface. This mechanism would provide time-dependent arrivals at arrays of surface dipoles. In principle, the first (Fresnel-zone) mechanism may occur at any boundary in the streaming potential coefficient, while the second mechanism requires the interface to provide a difference in both acoustic and electrokinetic properties.

In addition to the above body-wave coupling mechanisms, surface seismic waves must also be considered. Coupling due to surface waves is the third mechanism depicted in Fig. 1. Free-surface wave effects include the direct (compressional) wave and the surface/Rayleigh wave (ground-roll). Ground-roll effects, produced by dispersive Rayleigh waves, can be significant in shallow studies. Ground-roll is distinguished on seismic records by a high-amplitude and low-frequency waveform. The waves travel along the ground surface (their amplitudes decrease exponentially with depth) at a velocity of about 0.36 that of compressional waves (Dobrin \& Savit 1988). The two waves are characterized by time-distance traces which pass through the origin. In theory, the Rayleigh wave, by virtue of its large amplitude, should contain a large electric field due to a vertical mismatch of current across the free surface. The coupling mechanism is similar to that of the head wave but with a spreading wavefront propagating at a much reduced velocity and a capability to provide instantaneous (i.e. at time zero) electrokinetic coupling due to an at-surface vertical electric dipole.

\section{PREDICTIONS FROM ELECTROMAGNETIC MODELLING}

The geophysical signature of electrokinetic coupling will be linked to the behaviour of the vertical electric dipole ( VED). A VED may be generated at the surface (Rayleigh waves), or at depth directly beneath the shot-point (Fresnel-zone coupling) or as a result of head-wave propagation and coupling. The radiation from dipole sources in the presence of a conducting half-space is considered by Banos (1966). The various regions of validity of the formulas developed are summarized by Kraichman (1976). The behaviour of the fields associated with a buried VED was investigated using a numerical formulation of the problem. The code is based on a general solution (em1d written by Ki Ha Lee of Lawrence Berkeley Laboratories) for arbitrarily polarized electric and magnetic dipole sources in a layered earth. The results are shown for a unit dipole moment (1 A.m), a frequency of $80 \mathrm{~Hz}$ and a half-space resistivity of 100 ohm.m.

The horizontal electric field (E) observed in the near vicinity of a unit VED (1 A.m) from the surface to a depth of $50 \mathrm{~m}$ is shown in Fig. 2. The phase of all the fields undergoes a $180^{\circ}$ reversal about the source location and all amplitudes are symmetric. The at-surface dipole generates the largest $E$ field, centred above the point source. The field is spatially far more compact than those generated by a subsurface VED. In the case of a buried VED, the source location defines a local minimum whose amplitude is controlled by the depth of the source. Maximum at-surface $E$ fields occur, symmetrically, at an offset equal to half the depth to the source. Equally important is the phase reversal $\left(180^{\circ}\right)$ of the field oscillations occurring about the source location. The phase reversal is highly significant in that all other sources of electromagnetic radiation (natural and anthropogenic), which may interfere with the measurement, are distant and would appear as in-phase oscillations across the local scale of the measurement depicted. Subsurface interfaces may generate electrokinetic coupling directly beneath the shot-point. The problem is studied by extending the analysis of the VED to various depths and examining the signals obtained at various positions along the surface. For the analysis, 'virtual electrode separations' of vanishingly small length are assumed. Figure 3 shows the variation of the horizontal electric field at four offset positions $(2,4,10$ and $20 \mathrm{~m})$ for VED depths of between 1 and $50 \mathrm{~m}$. The moment of the VED remains constant (1 A.m) at all depths. At first sight, the response behaviour of individual offset locations shown in Fig. 3 may appear counterintuitive. Although, as noted previously, the maximum surface field amplitude is generated at an offset equal to one-half the source 
depth, for each specific location a maximum response is observed when the source depth is one-half the offset distance. From Fig. 3 it is evident that small offset locations provide the largest field amplitudes, particularly for near-surface VED sources, and the highest degree of depth sensitivity. For the example used (constant dipole moment with depth), field amplitudes from deeper sources of coupling (for example $>20 \mathrm{~m}$ ) will provide similar amplitudes at both near $(2 \mathrm{~m})$ and far $(20 \mathrm{~m})$ offsets. For a VED source at $50 \mathrm{~m}$ depth, the field amplitude at $2 \mathrm{~m}$ offset is reduced by less than one order of magnitude from that at an offset of $20 \mathrm{~m}$. Despite the fact that a unit dipole source has been used in the modelling, it is likely that shallow effects will produce the largest amplitudes and will dominate the voltage recordings.

\section{FIELD DATA ACQUISITION AND PROCESSING}

Predicted and observed electrokinetic voltages (Butler et al.1996; Mikhailov et al. 1997) can range from the millivolt to the sub-microvolt level. The technical specification of the acquisition system used (for example dynamic range) together with the noise reduction procedures employed ultimately control the degree to which the lower-level signals can be detected.

A variety of field data acquisition systems for electrokinetic measurements have been described in the literature. They range from modified and unmodified multichannel seismic systems (Thompson \& Gist 1993; Mikhailov et al. 1997) to purpose-built systems (Butler et al. 1996). Following trials with standard multichannel seismic acquisition systems, an eight-channel acquisition system was constructed to maintain the particular requirements of high-fidelity electric field measurements (high input impedance combined with high common-mode rejection). Signal conditioning comprises fixed and variable gain (200-1600) and a bandwidth from 10 to $1000 \mathrm{~Hz}$. Data are recorded at 16-bit resolution with a typical sampling frequency of 10 or $20 \mathrm{kHz}$, and the typical record length is 4000 data points. The system is battery powered, comprises a virtual earth, and can accept both electric dipole and geophone inputs. A standard inertia switch available for shallow seismics has been found to be the most accurate and reliable triggering method.

The removal of noise sources in individual (shot) voltage records is described in the context of electrokinetic observations by Butler et al. (1996) and Mikhailov et al. (1997). General aspects of electromagnetic noise are discussed by Szarka (1987). The removal of mains power harmonics is an important requirement in the processing of the recorded voltage data. The degree of mains harmonic processing required will be a function of the severity of the noise contamination and this is inevitably site-specific. It is advantageous to have a suite of mains harmonic removal techniques in place. Butler \& Russell (1993) discuss the techniques of block and sinusoid subtraction, which can both be applied. Both of these techniques work well at high sampling rates and in the presence of low to moderate levels of mains contamination (i.e. no amplitude modulation over the record length). In the case of severe mains contamination, these techniques can be supplemented by other techniques such as delay-line filtering (for example Szarka 1987). Apart from mains harmonic processing, the only other processing applied to the individual voltage records in the present study is that of low-pass (typically $<500 \mathrm{~Hz}$ ) filtering in cases of strong high-frequency noise contamination.

Stacking procedures from successive shot records should take into account the vagaries of other forms of noise contamination. Low-amplitude voltage records can be particularly susceptible to a number of problems, such as large-amplitude sferics, hammer and plate bounce. To overcome some of the more subtle forms of noise contamination, a graphical stacking procedure using a combination of visual stacking and monitoring of rms voltage levels has been implemented. As indicated by the electromagnetic modelling, recording arrays that exploit the phase asymmetry about the shot-point have been extensively used (the so-called split-spread).

Examples of dipole recording arrangements are shown in Fig. 4. All the configurations shown use $2 \mathrm{~m}$ dipoles which are symmetric and collinear about the shot-point. Other dipole lengths have also been used. Fig. 4(a) shows the simplest two-channel configuration with inner electrodes located at $\pm 0.5 \mathrm{~m}$ from the shot-point. This is referred to as the standard arrangement and is a component in all the other configurations. Fig. 4(b) shows an equatorial configuration using two perpendicular standard arrangements. This can obviously be extended to allow a full azimuthal arrangement. Fig. 4(c) shows a split-spread of non-overlapping, contiguous dipoles (10 channels shown) 
using $2 \mathrm{~m}$ separations. Fig. 4 (d) is an extension of the arrangement in the previous configuration and comprises overlapping dipoles (16 channels shown) at a separation of $1 \mathrm{~m}$. Such configurations are particularly important with regard to the identification of signal and noise. Over the spatial scale of a typical experiment, all external (for example atmospheric) and internal (for example mains components) sources of noise will appear as in-phase oscillations across surface dipoles. Any coupled response that generates a vertical electric dipole, either instantaneously or as a propagating wave, will produce out- of-phase oscillations across shot-symmetric dipoles (assuming a layered structure). In practice, the sign of one of each pair of channels is reversed so that 'signal' may be identified as an in-phase characteristic and 'noise' is characterized by its out- of-phase character. The symmetric voltage traces presented in this study all follow this convention.

As an example, consider the results of an eight-channel experiment that was undertaken in a particularly poor environment for coupling (dry marl). The results of stacking 50 shot records are shown as true amplitude traces in Fig. 5, with symmetric channels (one infilled, one line) jointly displayed. Dipole lengths were $2 \mathrm{~m}$ and centres were located at $\pm 1.5 \mathrm{~m}$ ( labelled 1, in Fig. 5), $\pm 2.5 \mathrm{~m}$ ( labelled 2), $\pm 3 \mathrm{~m}$ ( labelled 3) and $\pm 3.5 \mathrm{~m}$ ( labelled 4) from the shot-point. The recording time interval is from 160 to $200 \mathrm{~ms}$, where no signal is anticipated. It can be seen that the overall level of these residuals is typically less than $4 \mathrm{mV}$. Pairs of channels display strictly out-ofphase behaviour and the residual pattern is repeated across all pairs of dipoles. The residuals are formed due to incomplete noise cancellation, and, since all records are acquired simultaneously, the residuals appear as coherent traces across all channels. Such residuals can develop a wide variety of forms (i.e. frequency content), depending on site-specific noise contamination. The use of the split-spread, symmetric dipole arrangement allows such noise residuals to be rigorously identified.

\section{ELECTRODES AND DIPOLE LENGTH}

The majority of experiments were carried out with stainless- steel electrodes $0.5 \mathrm{~m}$ in length inserted about $0.4 \mathrm{~m}$ into the ground The electrokinetic voltage behaviour as a function of electrode type has been investigated many times. Fig. 6 shows the true voltage amplitudes that were obtained at one test site from three repeated experiments using the standard two-channel configuration with (i) stainless-steel (standard) electrodes; (ii) lead rods; and (iii) non-polarizing $\mathrm{Cu} / \mathrm{CuSO}_{4}$ electrodes. In each case the data shown are a stack of 10 repeat shots and symmetric channels (one infilled, one line) are jointly displayed.

Similar characteristics are observed in all three experiments, with the main, in-phase pulse observed between 5 and $12 \mathrm{~ms}$. The in-phase oscillation with an onset at about $30 \mathrm{~ms}$ appears to display a genuine asymmetry (in time) between the two channels. In most cases, the received voltages appear largely independent of electrode type, although electrode type can also determine ground contact resistance. In some environments, high electrode contact resistances have been found to degrade the data records. To improve data quality in arid environments, metal electrodes should be watered, or porous-pot electrodes should be used.

The horizontal electric field cannot be measured at a point. The gradient of the potential $(V)$ across two grounded electrodes is the routine method of measurement since $E=-\nabla V$. In practice, a very small electrode separation (for example $<1 \mathrm{~m}$ ) may result in a low signal-to-noise ratio, while a large separation may average the field gradients defining the spatial form of the field. Some form of compromise is inevitable. Prior to the application of multichannel experiments, investigations into the received voltage as a function of dipole length were carried out. The amplitude of noise components within the data, such as mains harmonics and sferics, scales linearly with dipole length. Electrokinetic 'signal', however, does not. Fig. 7 shows the behaviour resulting from increasing the dipole length when the position of the inner electrode (nearest to the shot) remains fixed. The data shown are the true amplitude voltages obtained symmetrically and collinearly about a shot location. The form of display follows that of Fig. 6 . The data shown are the resultant stack of 10 repeat shots with only mains harmonic suppression processing applied.

The symmetric voltage traces (i.e. to the left and right of the shot) are largely in-phase. The true amplitude results demonstrate that the received voltages are largely independent of dipole length when the position of the inner electrode remains at a fixed offset. The general type of behaviour shown in Fig. 7 has been observed repeatedly. The form of the voltage trace can vary with dipole length. This is often observed in the initial 10 or $20 \mathrm{~ms}$ of recording. The behaviour is likely to be connected with the relationship between spatial gradients and the inevitable 
averaging effect of longer dipoles. The important result is that it is the inner electrode (i.e. that nearest the shot) that controls the amplitude and character of the received voltage.

\section{TWO-CHANNEL EXPERIMENTS}

The two-channel approach to electrokinetic experiments forms the basis of a commercial geophysical instrument intended for hydrogeological investigations (Millar 1995). The concept is that the voltage returns observed on the two dipoles can be interpreted entirely by electrokinetic coupling vertically beneath the shot-point (Fresnel-zone coupling). In principle this mechanism provides a vertical sounding capability, since coupling should occur at the oneway travel-time of the acoustic wave. The basic investigation into the coupling then concerns the time-dependent behaviour of the voltages that are recorded by symmetrical surface dipoles and the degree to which they are in-phase (allowing for the sign reversal of one of each pair of dipoles).

Although conceptually simplistic, the two-channel approach offers a rapid summary of:

(1) the existence of electrokinetic coupling at a particular location;

(2) its amplitude;

(3) the degree of in-phase (symmetric) voltage behaviour;

(4) the time duration of any coupling behaviour.

In the standard arrangement (Fig. 4a), contributions from wave-moveout effects should be minimized.

Sandstone environments have been found to provide early time coupling in the millivolt range. The first example is shown in Fig. 8: a sounding undertaken entirely on sandstone near a borehole indicating a water-table depth of $44 \mathrm{~m}$. Fig. 8 shows the first $50 \mathrm{~ms}$ of data from the first six shots obtained from the standard two-channel symmetric deployment. The first shot record is shown by the two dashed traces. The voltage oscillations are due to electrode shake and can clearly be identified by large-amplitude oscillations which decay with time. The data obtained from the next five shots, following more rigid electrode insertion, are shown by the heavy solid and dotted traces. Even the small number of records shown indicate that symmetrical, in-phase behaviour can be observed over a substantial portion of the time interval. It is also evident that, for all realistic acoustic velocities (and one-way traveltimes), interfaces prior to the main water table must be associated with the behaviour observed.

Two-channel operation can be extended to a contiguous profiling mode in which each sounding centre is separated by $5 \mathrm{~m}$. In this mode, the repeatability of the voltage characteristics is examined sequentially along a profile. In many cases, particularly those involving low amplitude (for example $0.1 \mathrm{mV}$ ) levels, very little repeatability is observed. Fig. 9 shows an example of one channel (for example a dipole $0.5-2.5 \mathrm{~m}$, to the right of a shot obtained every $5 \mathrm{~m}$ along a profile. The first $40 \mathrm{~ms}$ of the trace-normalized stack results are shown. The data were obtained along a dry sequence of gravel/sands ( $20 \mathrm{~m}$ thick) overlying a sandy marl. It is evident in the results that the degree of spatial repeatability is very low in this dry environment.

At-surface sand units typically provide large early time oscillations that are spatially repeatable. Fig. 10 shows an example of standard two-channel behaviour along a short profile where the geological sequence comprises an at-surface, saturated sand $(0-2 \mathrm{~m})$ overlying a sequence of clays $(2-20 \mathrm{~m})$ above chalk. The first $10 \mathrm{~ms}$ of data shown in Fig. 10 are true amplitudes, with voltages to the left of each shot shown as infilled traces and voltages to the right of each shot shown as single lines. It is clear that, over the first $10 \mathrm{~ms}$, in-phase behaviour is observed and the spatial repeatability is high. An instantaneous positive (to the right) potential of several millivolts is followed by a rapid negative swing which is completed within $2 \mathrm{~ms}$. This large-amplitude instantaneous behaviour appears to be connected with the saturated sand unit; however, the apparent high frequency of the oscillation remains to be explained. It can also been seen that the two symmetric channel amplitudes undergo a change in character from a distance of $20 \mathrm{~m}$ onwards.

The assessment of the electrokinetic site characteristics using two-channel (standard) operation has been carried out over a wide variety of geological environments. Although a wide variety of amplitudes and characteristics have been observed, the following key features have emerged. 
(1) The largest amplitude oscillations occur in the first 10-20 ms of recording. The coupling is observed in both dry and saturated environments.

(2) Using hammer sources, received voltages range from a maximum excursion of about $15 \mathrm{mV}$ to small values (for example $1 \mathrm{mV}$ ). The smaller voltage ranges have been observed, typically, in clay and marl environments. The smaller voltage returns are not generally associated with strong in-phase behaviour.

(3) The same voltage effects are observed when using metal-hammer/metal-plate and metal hammer/nylon -plate combinations.

(4) The spectral characteristics of the voltages during the first tens of milliseconds show a similar dominant frequency to acoustic data records (electrodes replaced by $10 \mathrm{~Hz}$ vertical geophones). For hammer sources this tends to range from 50 to $100 \mathrm{~Hz}$ with a dominant component often at about $70 \mathrm{~Hz}$.

The two-channel standard operation has often been extended using the azimuthal arrangement (Fig. 4b). Results that are independent of orientation and results that show a clear dependence on azimuth have both been observed. Voltage data that display a dependence on azimuth over an area of just $5 \mathrm{~m}^{2}$ are likely to require interpretation in terms of shallow anisotropic properties.

\section{MULTI-CHANNEL EXPERIMENTS}

When electrokinetic observations are extended laterally as shown in Fig. 4, questions of spatial scale and the precise arrangement of surface dipoles are raised. Although some experiments have extended dipoles to offset distances of $>10 \mathrm{~m}$ from the shot-point, the majority of experiments required very detailed measurements, often with overlapping $1 \mathrm{~m}$ and $2 \mathrm{~m}$ dipoles, in close proximity to the shot. Fig. 11 shows the true-amplitude, symmetric voltage traces from an experiment which used $2 \mathrm{~m}$ dipoles with centres at $\pm 1.5, \pm 2.5$ and $\pm 3.5 \mathrm{~m}$ (inner electrodes at offsets of $0.5,1.5$ and $2 \mathrm{~m}$ from the shot-point). The final dipole (channel 4 in Fig. 11) comprised a 4 $\mathrm{m}$ dipole with a centre at $3 \mathrm{~m}$ (inner dipole at $1 \mathrm{~m}$ ). A dramatic fall-off in amplitude between the innermost dipoles (channels 1) and subsequent dipoles (channels 2 and 3), with offsets increased by 1 and $1.5 \mathrm{~m}$, can be observed. Channel 4 ( $4 \mathrm{~m}$ dipole) has an inner-electrode offset increased by only $0.5 \mathrm{~m}$ from that of channel 1 and even over this distance time- dependent (moveout) behaviour of the characteristic voltage trace can be observed.

The next example is in an area where the mapped geology is thin Boulder Clay $(<10 \mathrm{~m})$ overlying Sherwood Sandstone. A full-scale refraction experiment (a spread length of $100 \mathrm{~m}$ ) was carried out to establish acoustic velocities. The end shots proved competent rock with velocities in the range $2890-3203 \mathrm{~m} \cdot \mathrm{s}^{-1}$ at depths between 16 and $25 \mathrm{~m}$. This is overlain by a layer of velocity $800 \mathrm{~m} \cdot \mathrm{s}^{-1}$ which is in turn covered by unconsolidated material (velocity of 365-560 m.s ${ }^{-1}$ ) about $5 \mathrm{~m}$ thick. A joint electrokinetic/acoustic moveout experiment was conducted in the centre of the refraction profile. A split-spread using dipole length of $50 \mathrm{~cm}$ and offsets from 0.5 to $6 \mathrm{~m}$ was used. The voltage data are shown as normalized wiggle traces in Fig. 12(a). An onset time of $4 \mathrm{~ms}$ is used to avoid the necessity of normalizing by the large early time oscillation that occurs on all traces. The results show that at locations both less than and greater than a standard dipole centre of $1.5 \mathrm{~m}$, moveout effects can be detected in all the main voltage oscillations. Although the data show a degree of coherence (in-phase behaviour) about the shot-point, some asymmetry is also evident, with faster apparent velocities observed at positive offsets. Other forms of presentation also reveal that the weaker voltage oscillations at later times (towards $100 \mathrm{~ms}$ ) display moveout at similar velocities to the earlier voltage oscillations. The apparent velocities are low and range from $350 \mathrm{~m} \cdot \mathrm{s}^{-1}$ to a maximum of about $500 \mathrm{~m} \cdot \mathrm{s}^{-1}$.

The acoustic experiment duplicated the electrokinetic experiment, with $10 \mathrm{~Hz}$ vertical geophones placed at $0.5 \mathrm{~m}$ intervals. The acoustic data are shown as normalized wiggle traces in Fig. 12(b). The first, high-frequency arrival observed on the traces is the air-wave from the hammer/plate combination with a velocity of $330 \mathrm{~m} \cdot \mathrm{s}^{-1}$. Subsequent arrivals, including the direct wave, are of similar low velocity and do not exceed $400 \mathrm{~m} \cdot \mathrm{s}^{-1}$. Below the airwave, the first arrival displays non- linear moveout as the shot-point is approached (i.e. at offsets $<3 \mathrm{~m}$ ). Towards later times, a much slower arrival is detected (about $140 \mathrm{~m} \cdot \mathrm{s}^{-1}$ ). It is apparent that low-velocity surface-wave effects 
seem to dominate the acoustic records. Such effects are less conspicuous in the voltage recordings of Fig. 12( b). Similar effects are reported by Butler et al. (1996).

A further example comes from an experiment conducted at the Gore Sand River in southern Zimbabwe. The Gore Sand River channel (a former drainage course) is incised into crystalline basement rocks (largely granite). The river is about $15 \mathrm{~m}$ wide and infill is thought to be about $6 \mathrm{~m}$ thick. The water table is estimated to be about $0.5 \mathrm{~m}$ below the surface. Fig. 13 is a summary of the typical dual-field moveout behaviour observed in the immediate vicinity of a shot-point. The experiment is highly detailed (E-field dipole lengths of $1 \mathrm{~m}$ ), with both sets of measurements obtained at intervals of $50 \mathrm{~cm}$. $10 \mathrm{~Hz}$ vertical geophones and a data acquisition rate of $20 \mathrm{kHz}$ were used. The two sets of results have been treated identically using individual trace normalization (to +1 and -1 ) across the 100 ms time window shown.

The seismic data, contoured in the left frame, display time- offset gradients out from the shot-point, and these gradients define apparent velocities of the propagating waves. There is a high degree of symmetry in the behaviour about the shot- point. Standard seismic refraction analysis of the first arrivals indicates a three-layer velocity sequence of 180,380 and $1000 \mathrm{~m}^{-1} \mathrm{~s}^{-1}$ with interface depths of 0.6 and $1.3 \mathrm{~m}$. Although there are indications of superimposed effects following the first arrival (first negative/positive-going pulse), two further arrivals (>40 ms) can be identified (on the flanks of the image) having estimated moveout velocities of 400 and 1300 m.s ${ }^{-1}$. These velocities are consistent with head waves propagating along the two refractor interfaces. A low-velocity feature with an onset time of about $26 \mathrm{~ms}$ in the centre may be a shallow reflection hyperbola.

The voltage data, contoured in the right frame of Fig. 13, appear to reflect the behaviour of the seismic data to some degree. The first and largest oscillation has an onset delay of several (3-4) milliseconds and can be traced to an offset of $7 \mathrm{~m}$ with a velocity of between 300 and $400 \mathrm{~m} \cdot \mathrm{s}^{-1}$. The apparent moveout velocity of the three coherent oscillations (at times $>30 \mathrm{~ms}$ ) is between 200 and $350 \mathrm{~m} \cdot \mathrm{s}^{-1}$ and they possess the same dominant frequency (about $90 \mathrm{~Hz}$ ) as the seismic data. All the voltage oscillations appear to be associated with electrokinetic coupling due to the passage of horizontally propagating acoustic waves.

The general form of the voltage moveout behaviour shown in Figs 12 and 13 has been repeated in a number of experiments over a wide variety of environments. The largest amplitude oscillations, typically occurring in the first few tens of milliseconds, display moveout behaviour. These are followed, at later times, by a sequence of further oscillations which decrease in amplitude and again display moveout effects. The apparent velocity of these oscillations is invariably low (typically $<500 \mathrm{~m} \cdot \mathrm{s}^{-1}$ ).

\section{SUMMARY AND CONCLUSIONS}

This study has examined some general observational characteristics of electrokinetic coupling based on experiments carried out over a wide range of hydrogeological environments. The effects that are observed are pervasive, although amplitudes and character can vary considerably. The effects observed appear to be associated with both the unsaturated and saturated zones.

Recent theoretical predictions of the coupled elastic and electromagnetic wave equations have been discussed. It has been noted that an understanding of the characteristic radiation patterns from subsurface vertical electric dipoles is required. An electromagnetic modelling study, using unitary vertical electric dipoles, has provided information on the depth sensitivity of arrays of surface receivers. Small offset locations provide the largest horizontal electric field amplitudes and the highest degree of sensitivity. The modelling also suggests that shallow effects will produce the largest amplitudes and will dominate the voltage recordings by several orders of magnitude.

Recording arrays that rigorously exploit the phase asymmetry about the shot-point have proved to be advantageous in the identification and separation of signal and noise effects. Electrokinetic voltage records at low voltage levels $(<0.1 \mathrm{mV})$ are particularly susceptible to inconsistent shot effects. Graphical stacking procedures which allow for such subtle noise effects have proved to be more effective than brute-force schemes. Following repeated trials in a number of environments, the received voltages have been found to be largely independent of electrode type. Rigid insertion (to avoid electrode shake) and low contact resistance are the 
main requirements, irrespective of electrode type. Although noise components scale with dipole receiver length, the received voltages do not. An important observation is that it is the inner electrode of each dipole (i.e. that nearest the shot-point) that controls the amplitude, and, to some extent, the character of the received voltage.

Relatively simple two-channel experiments have been described that offer rapid summaries of the existence of electro-kinetic coupling at a particular location, its amplitude, the degree of in-phase (symmetric) voltage behaviour, and the time duration of any coupling behaviour. The largest amplitude oscillations occur in the first 10$20 \mathrm{~ms}$ of recording. Using hammer sources, received voltages range from a maximum excursion of about $15 \mathrm{mV}$ to small values (for example $0.1 \mathrm{mV}$ ). The smaller voltage ranges have been observed, typically, in clay and marl environments. The smaller voltage returns are not generally associated with in-phase behaviour. The spectral characteristics of the voltages during the first tens of milliseconds show a similar dominant frequency to those from acoustic data (for example a peak frequency of $50-100 \mathrm{~Hz}$ ).

Multi-channel experiments involving joint electric and acoustic measurements have been described. The rapid spatial decay of voltage amplitude with increasing offset, predicted by the VED modelling, is a persistent feature of the data obtained. The majority of such experiments have indicated that shot-symmetric voltages which display moveout are the dominant waveforms. Such moveout behaviour has been observed to times in excess of 200 $\mathrm{ms}$, although this is not to say that instantaneous arrivals due to Fresnel-zone coupling do not occur. Such behaviour has been observed in the experiments of Butler et al. (1996) and Mikhailov et al. (1997). At the present time there is a paucity of firm theoretical predictions concerning the nature of electrokinetic coupling. These are required to add substance to the interpretation of the extended experimental observations presented here. The main predictions that are available concern the roles of vertical ( $P$-wave) and horizontal (refracted head-wave, etc.) effects, as discussed previously.

The main lack of predictions concerns the role of electrokinetic coupling along the free surface (i.e. the role of the horizontally propagating direct and Rayleigh waves), which is likely to be highly significant. Butler et al. (1996) discuss anticipated direct $P$-wave effects, but no publications have dealt with the slower surface-wave effects. According to Pride (1997, personal communication) there should be a very large E-field inside a surface wave (the boundary being the free surface) that would be recorded as the wave passes surface dipole antennas. The concept is one of an expanding circular wavefront creating a vertical mismatch in streaming potential current (due to the interface), which would act as a circular region of vertical dipoles. Although the low moveout velocities of much of our voltage data are compatible with the slow acoustic propagation of Rayleigh and direct waves, the expectation is that such waves would trace back to time zero at the shot-point location. This behaviour is not observed. The moveout behaviour observed appears to be more readily interpreted by horizontally propagating refracted head-wave effects.

\section{ACKNOWLEDGMENTS}

This work was partly supported by the Department for International Development ( TDR Project R6232) and by the EEC through INCO-DC Project Number 950176. Thanks are also due to Roger Peart for his source properties (the perfect hammer swing) and for many helpful discussions. This paper is published with the approval of the Director, British Geological Survey (NERC).

\section{REFERENCES}

Ahmed, M.U., 1964. A laboratory study of streaming potentials, Geophys. Prospect., 12, 49-64.

Banos, A., 1966. Dipole Radiation in the Presence of a Conducting Half-Space, Pergamon Press, New York, NY.

Biot, M.A., 1956. Theory of propagation of elastic waves in a fluid saturated porous solid, I, Low-frequency range, J. acoust. Soc. Am., 28, 168-178. 
Biot, M.A., 1962. Mechanics of deformation and acoustic propagation in porous media, J. appl. Phys., 33, 1482-1498.

Bockris, J. \& Reddy, A.K.N., 1970. Modern Electrochemistry, Vol. 2, Plenum Press, New York, NY.

Broding, R.A., Buchanan, S.D. \& Hearn, D.P., 1963. Field experiments on the electroseismic effect, IEEE Trans. Geoscience Electronics, GE-1, 23-31.

Butler, K.E. \& Russell, R.D., 1993. Subtraction of powerline harmonics from geophysical records, Geophysics, 58, 898903.

Butler, K.E., Russell, R.D., Kepic, A.W. \& Maxwell, M., 1996. Measurement of the seismoelectric response from a shallow boundary, Geophysics, 61, 1769-1778.

Dobrin, M.B. \& Savit, C.H., 1988. Introduction to Geophysical Prospecting, McGraw-Hill Book Co., New York, N.Y.

Fitterman, D.V., 1978. Electrokinetic and magnetic anomalies associated with dilatant regions in a layered earth, J. geophys. Res., 83, 5923-5928.

Gaskarov, I.V. \& Parkhomenko, E.I., 1974. The seismoelectric effect in rocks and preconditions for its application in geological prospecting work, Izvestiya, Acad. of Sciences, USSR, Phys. Solid Earth, 1, 71-74.

Haartsen, M.W., Dong, W. \& Toksoz, M.N., 1998. Dynamic streaming currents from seismic point sources in homogeneous poroelastic media, Geophys. J. Int., 132, 256-274.

Ishido, T. \& Mizutani, H., 1981. Experimental and theoretical basis of electrokinetic phenomena in rock-water systems and its applications to geophysics, J. geophys. Res., 86, 1763-1775.

Ivanov, A.G., 1939. Effect of electrization of earth layers by elastic waves passing through them, Doklady Akademii Nauk SSSR, 24(1), 41-45 (in Russian).

Kraichman, M.B., 1976. Handbook of Electromagnetic Propagation in Conducting Media, Second Printing, US Government Printing Office, Washington.

Long, L.T. \& Rivers, W.K., 1975. Field measurement of the electroseismic response, Geophysics, 40, 233-245. Martner, S.T. \& Sparks, N.R., 1959. The electroseismic effect, Geophysics, 24, 297-308.

Milkhailov, O.V., Haartsen, M.W. \& Toksoz, M.N., 1997. Electroseismic investigation of the shallow subsurface: Field measurements and numerical modeling, Geophysics, 62, 97-105.

Millar, J., 1995. A source of life made commercial, Physics World, Oct. 1995, 22-23.

Murty, Y.S., 1985. First results on the direct detection of groundwater by seismoelectric effect-a field experiment, Bull. Austr. Soc. Expl. Geophys., 16, 254-255.

Neev, J. \& Yeatts, F.R., 1989. Electrokinetic effects in fluid-saturated poroelastic media, Phys. Rev., B40, 9135-9141.

Overbeek, J.Th.G., 1952. Electrochemistry of the double layer, in Colloid Science, Vol. 1, Irreversible Systems, pp. 115-193, ed. Kruyt, H.R. Elsevier, New York, NY.

Parkhomenko, E.I., 1971. Electrification Phenomena in Rocks, Plenum Press, New York-London.

Parkhomenko, E.I. \& Gaskarov, I.V., 1971. Borehole and laboratory studies of the seismoelectric e ect of the second kind in rocks, Izvestiya Acad. of Sciences, USSR, Phys. Solid Earth, 9, 663-666. 
Pride, S.R., 1994. Governing equations for the coupled electro-magnetic and acoustics of porous media, Phys. Rev., B50, 15 678-15696.

Pride, S.R. \& Haartsen, M.W., 1996. Electroseismic wave properties, J. acoust. Soc. Am., 100, 1301-1315.

Pride, S.R. \& Morgan, F.D., 1991. Electrokinetic dissipation induced by seismic waves, Geophysics, 56, 914-925.

Russell, R.D., Butler, K.E., Kepic, A.W. \& Maxwell, M., 1997. Seismoelectric exploration, The Leading Edge, SEG, 16, 1611-1615.

Szarka, L., 1987. Geophysical aspects of man-made electromagnetic noise in the earth-a review, Surv. Geophys., 9, 287-318.

Thompson, R.R., 1936. The seismic-electric e ect, Geophysics, 1, 327-335. Thompson, A.H. \& Gist, G.A., 1993. Geophysical applications of electrokinetic conversion, The Leading Edge, SEG, 12, 1169-1173. 


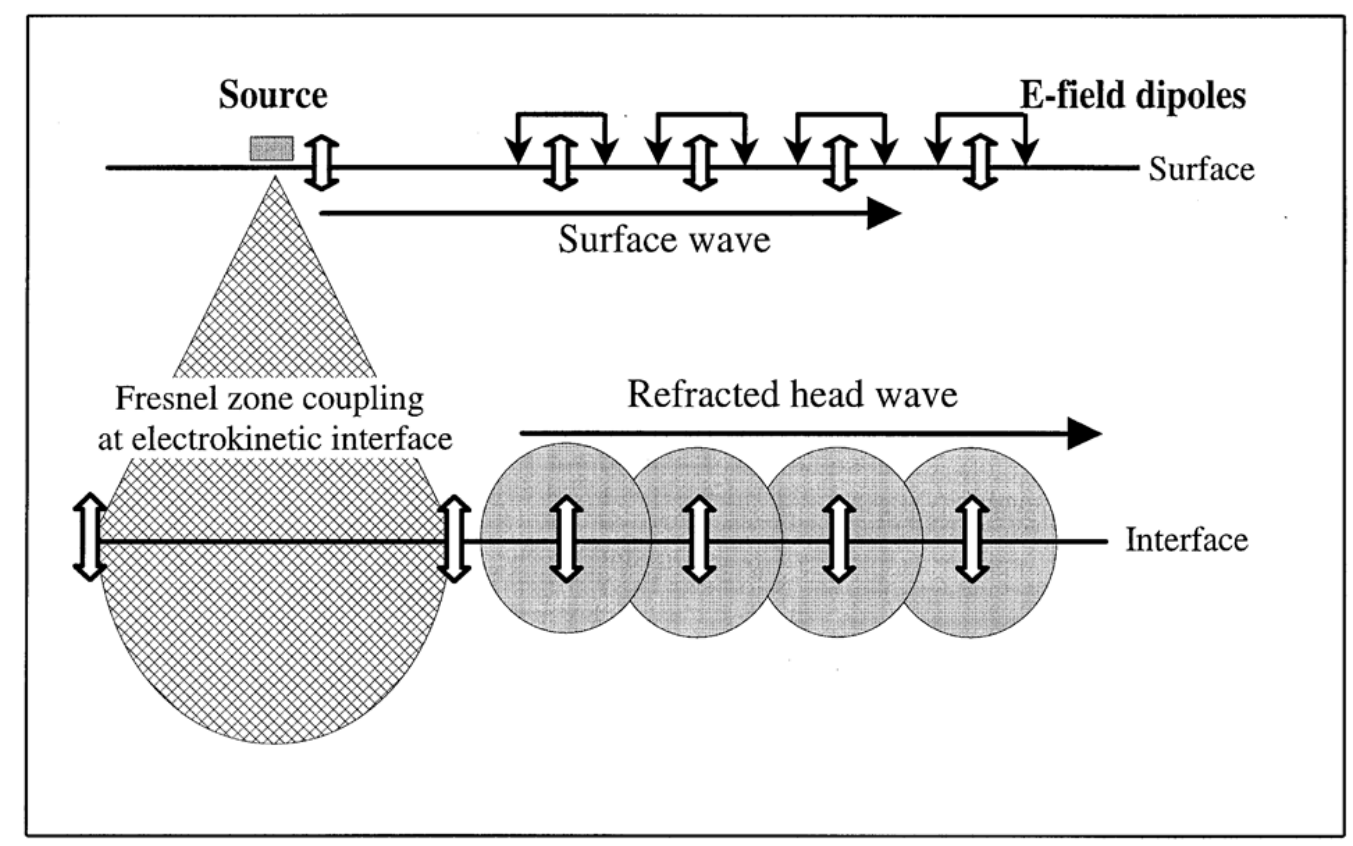

Figure 1. Schematic diagram of three possible mechanisms of electrokinetic coupling due to an acoustic source. All three mechanisms may produce an oscillating vertical electric dipole (double arrows) at an interface in streaming potential. (1) Surface-wave coupling and propagation. (2) Fresnel-zone coupling. (3) Refracted headwave coupling and propagation. 


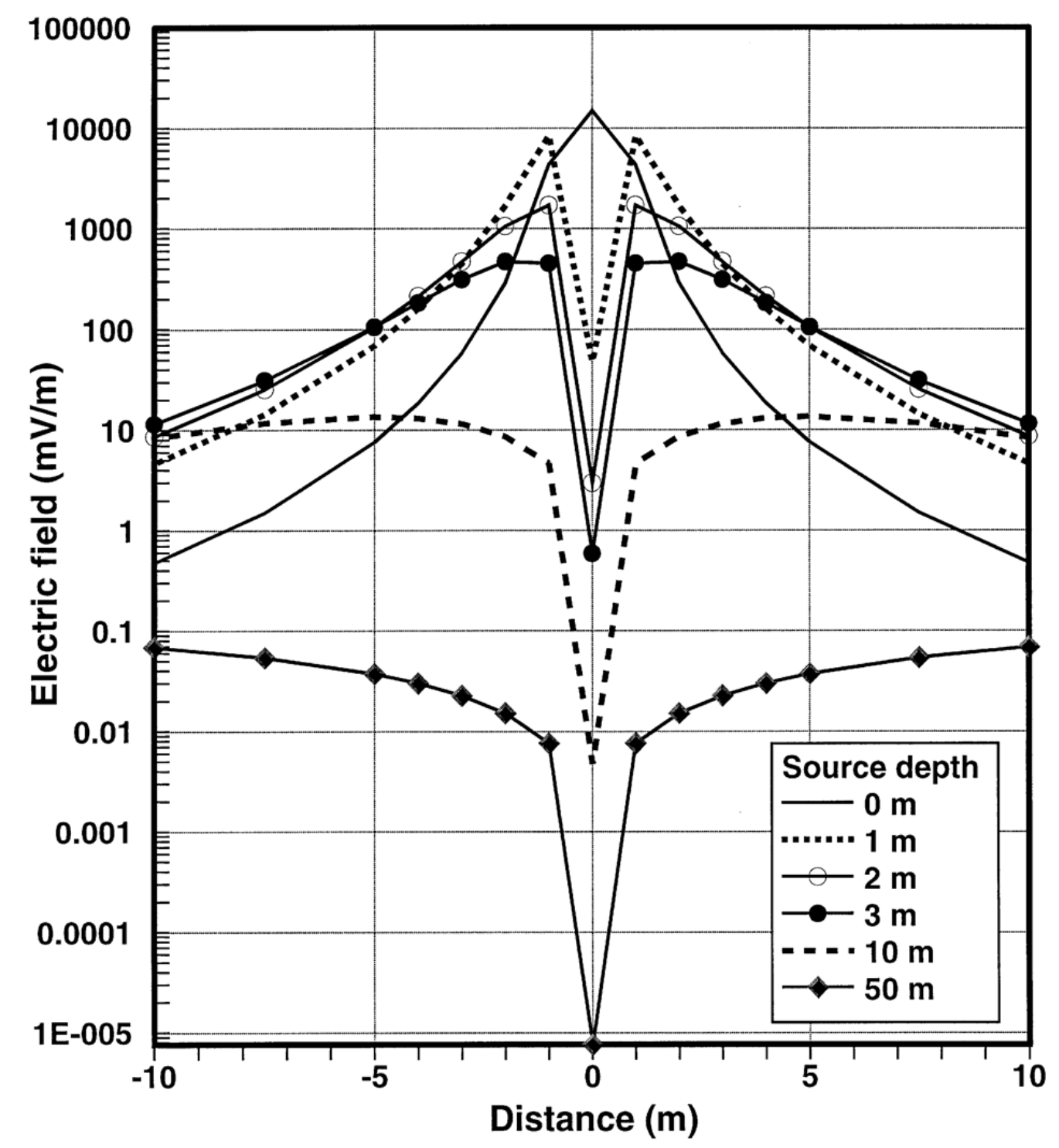

Figure 2. Variation of the horizontal electric field at the surface due to a vertical electric dipole source at the origin. Six depths of the dipole source are shown, including the surface. The dipole moment is 1 A.m, the frequency is $80 \mathrm{~Hz}$, and the calculation is done for a half-space of $100 \mathrm{Vm}$ and a dielectric constant of unity. 


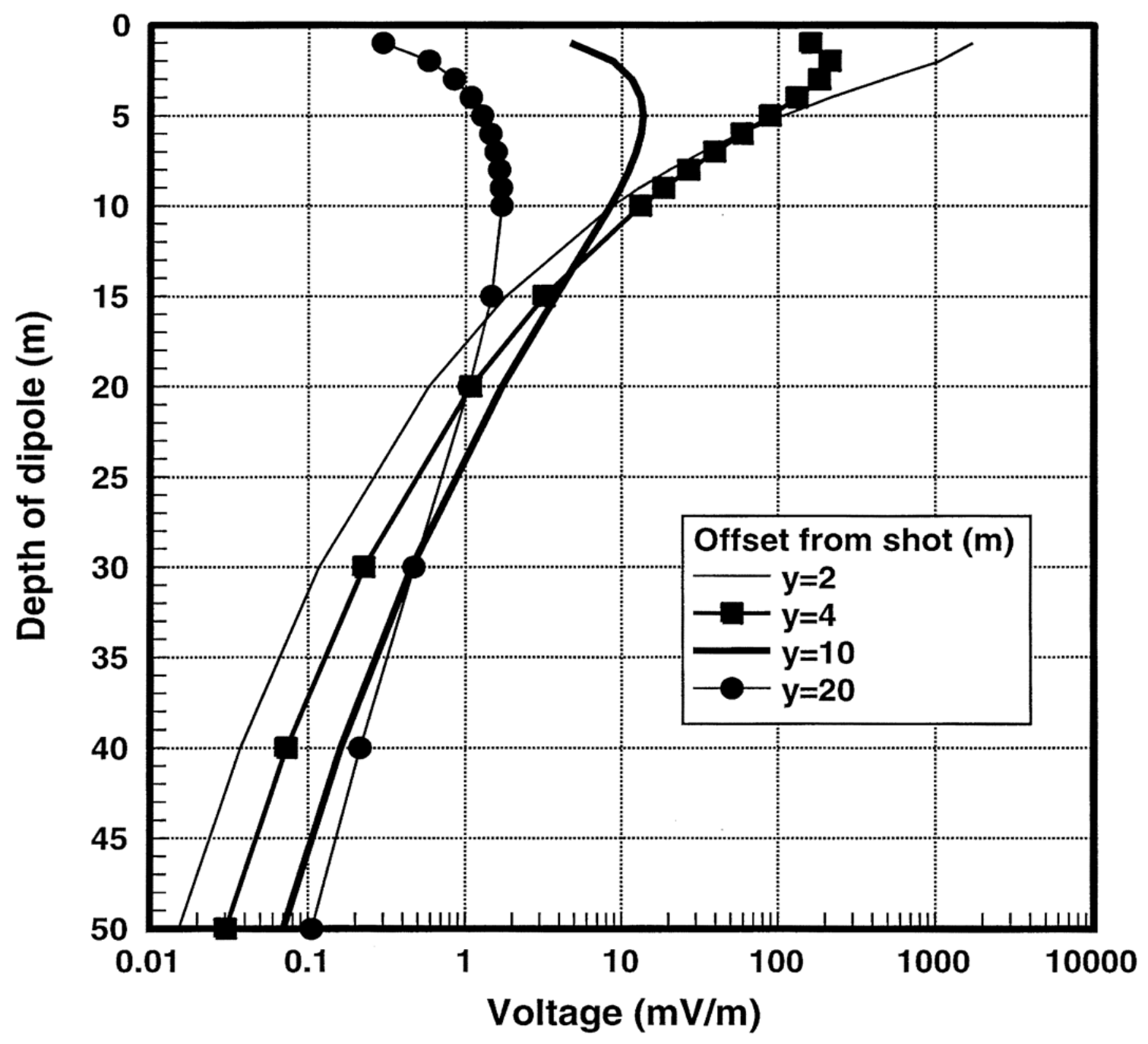

Figure 3. Variation of the amplitude of the horizontal electric field generated by a buried vertical electric dipole at depths between 1 and $50 \mathrm{~m}$. Four measurement locations are shown at offsets of 2, 410 and $20 \mathrm{~m}$ from the horizontal location of the dipole. The dipole moment is $1 \mathrm{~A} . \mathrm{m}$ at all depths, the frequency is 80 $\mathrm{Hz}$, and the calculation is done for a half-space of $100 \mathrm{~V} \mathrm{~m}$ and a dielectric constant of unity. 

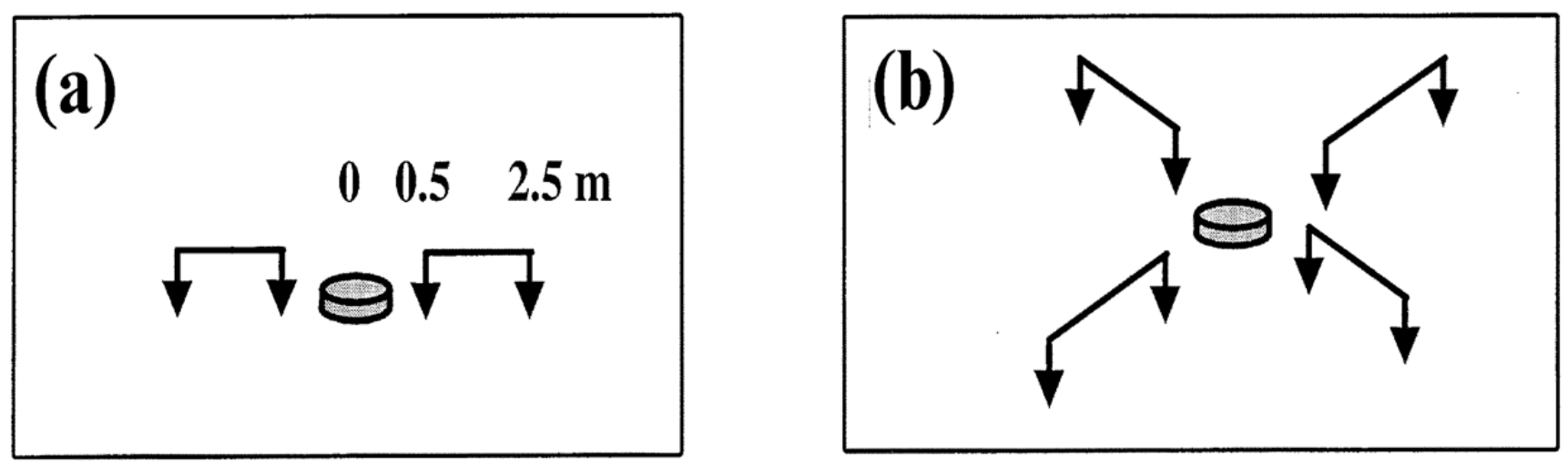

(c)

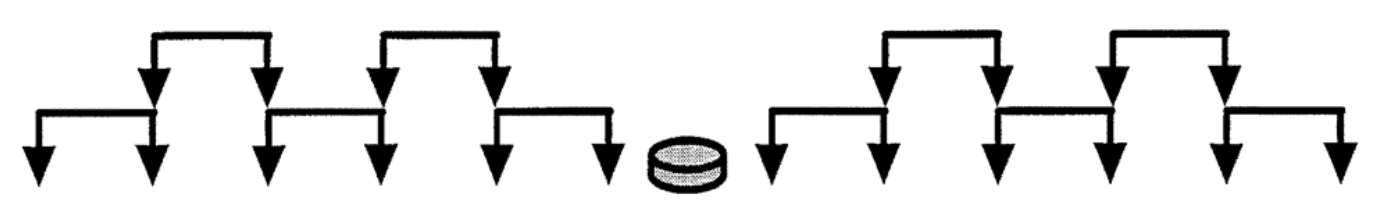

(d)

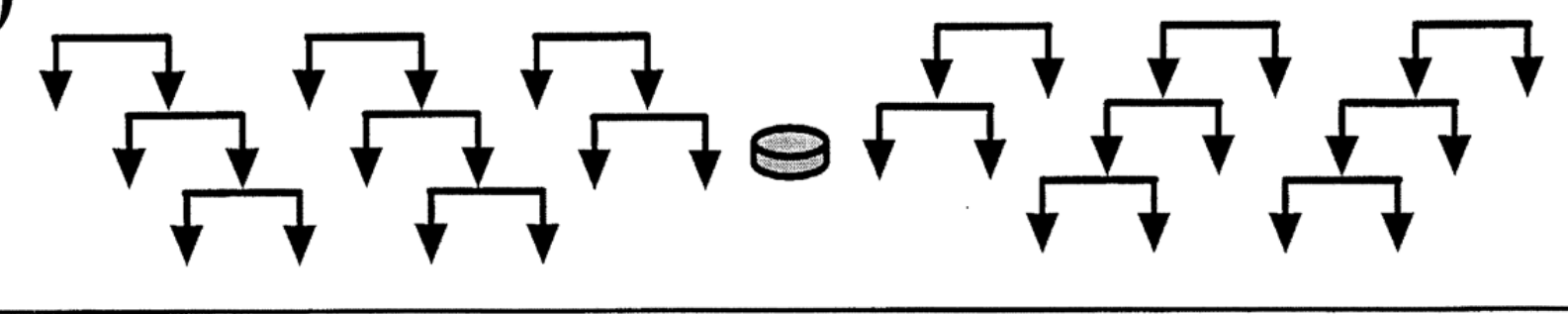

Figure 4. Examples of grounded-dipole recording arrangements. All four configurations shown use $2 \mathrm{~m}$ dipoles which are symmetric and collinear about the shot-point. (a) two-channel configuration. (b) Equatorial configuration (four channels). (c) Non-overlapping, contiguous dipole profile ( 10 channels shown). (d) Overlapping dipole profile (16 channels shown). 


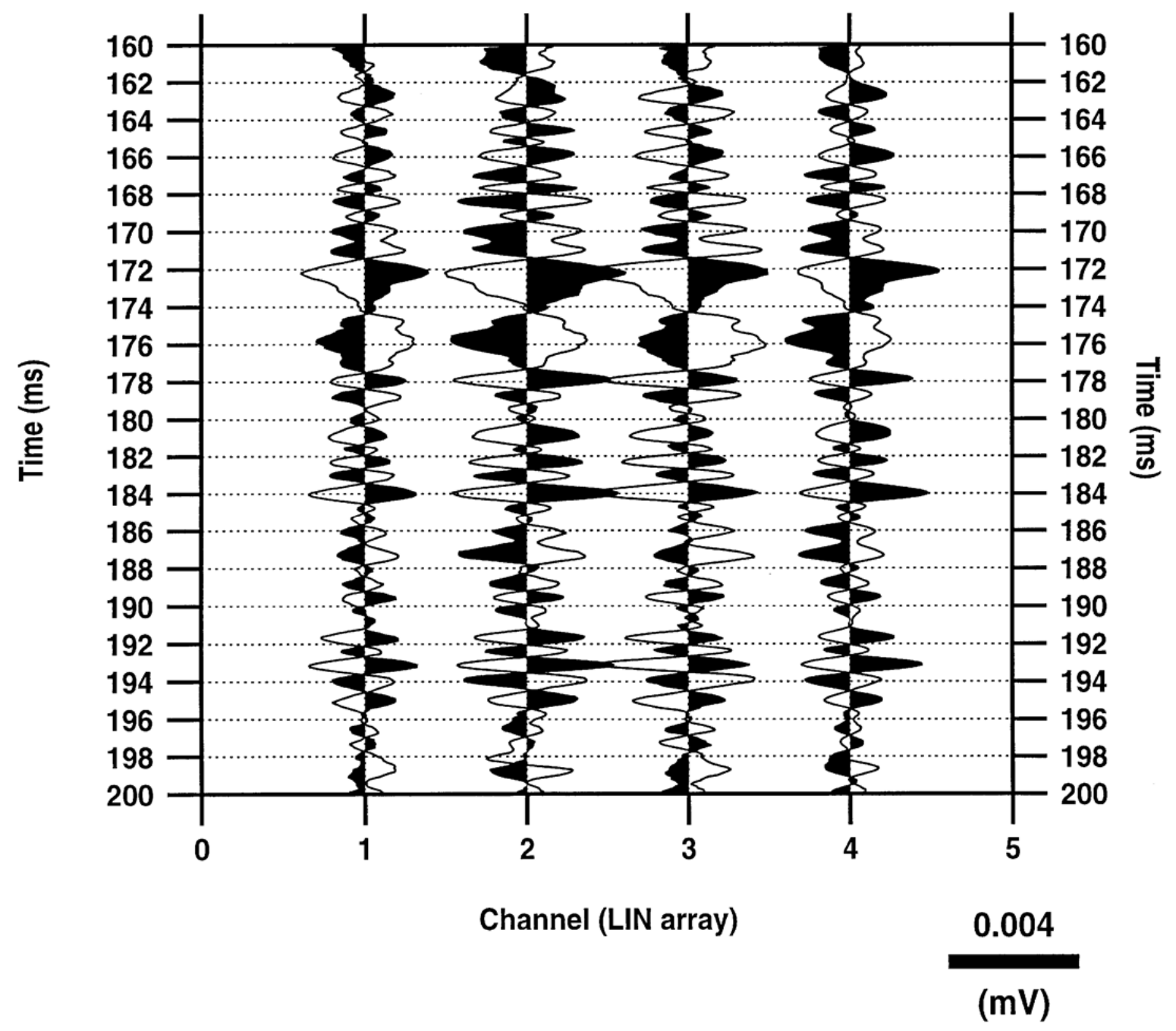

Figure 5. Example of an eight-channel recording using $2 \mathrm{~m}$ dipoles in a split-spread profile. Dipole centres are located at $\pm 1.5 \mathrm{~m}$ ( labelled 1 ), $\pm 2.5 \mathrm{~m}$ ( labelled 2), $\pm 3 \mathrm{~m}$ ( labelled 3 ) and $\pm 3.5 \mathrm{~m}$ ( labelled 4) from the shotpoint. Symmetric channels are jointly displayed as a single line trace and an infilled trace. 


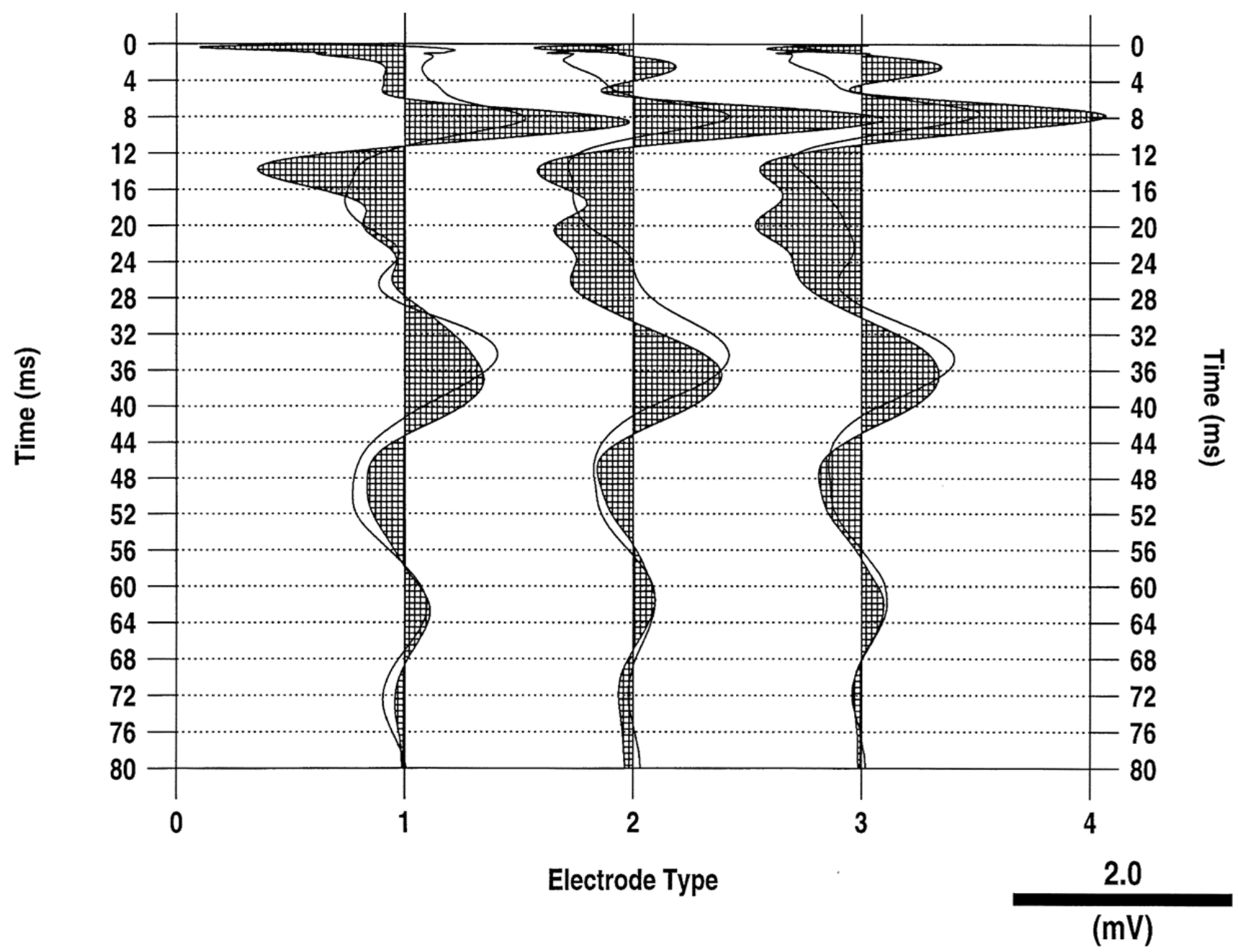

Figure 6. Comparison of results obtained with three different types of electrodes. Two-channel recording using the standard configuration. Symmetric channels are jointly displayed as a single line trace and an infilled trace. Electrode type 1 is standard stainless steel. Type 2 is lead rod. Type 3 is non-polarizing copper/copper sulphate. 


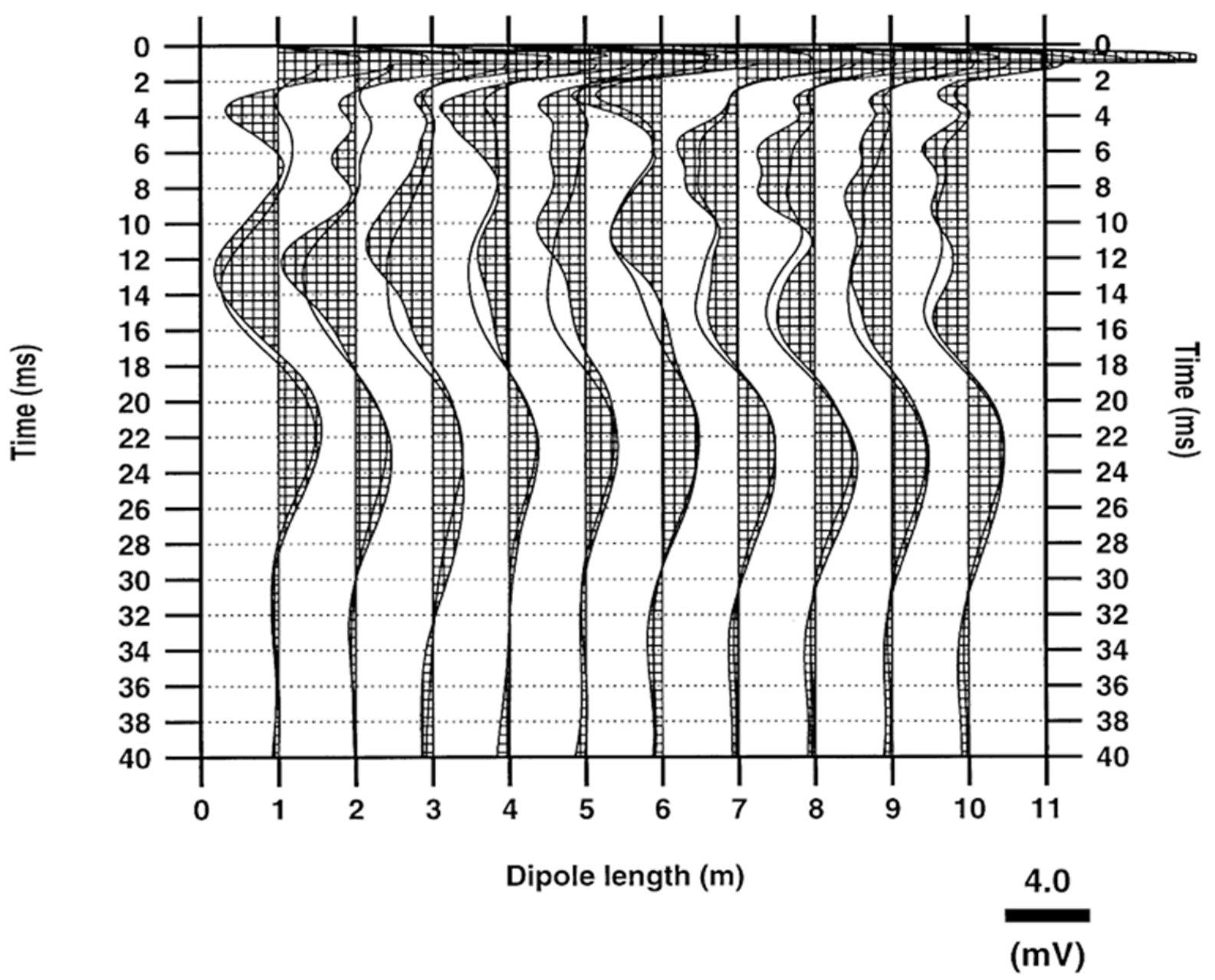

Figure 7. Comparison of results obtained by keeping the inner electrode at a fixed location ( $0.5 \mathrm{~m}$ from the shot) and expanding the outer electrode position to provide dipole lengths from 1 to $10 \mathrm{~m}$. Symmetric channels are jointly displayed as a single line trace and an infilled trace. 


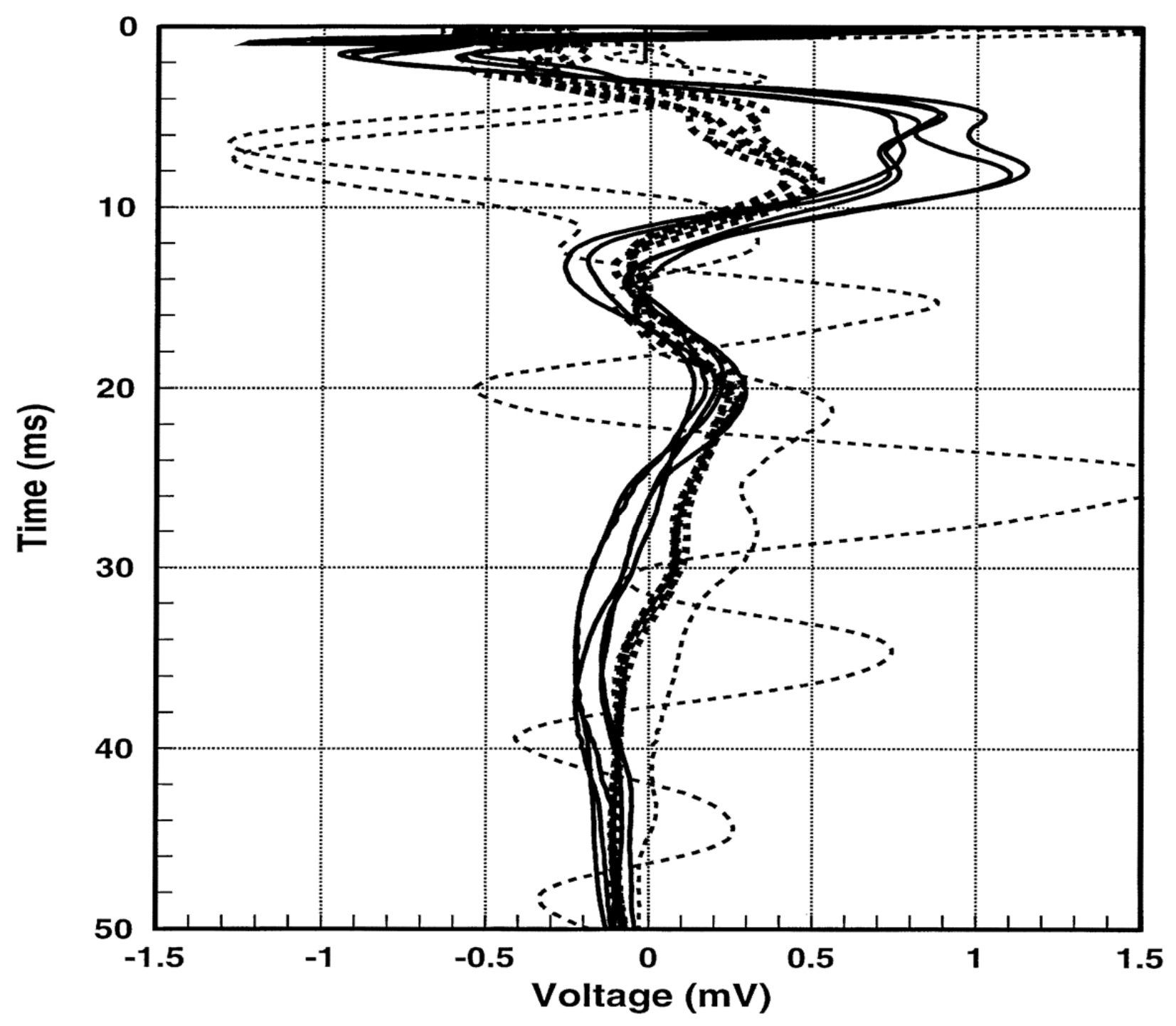

Figure 8. Six individual shot records obtained using the standard two-channel configuration. The first shot record is due to electrode shake (dashed lines). The following five records are jointly displayed as single and dotted lines. 


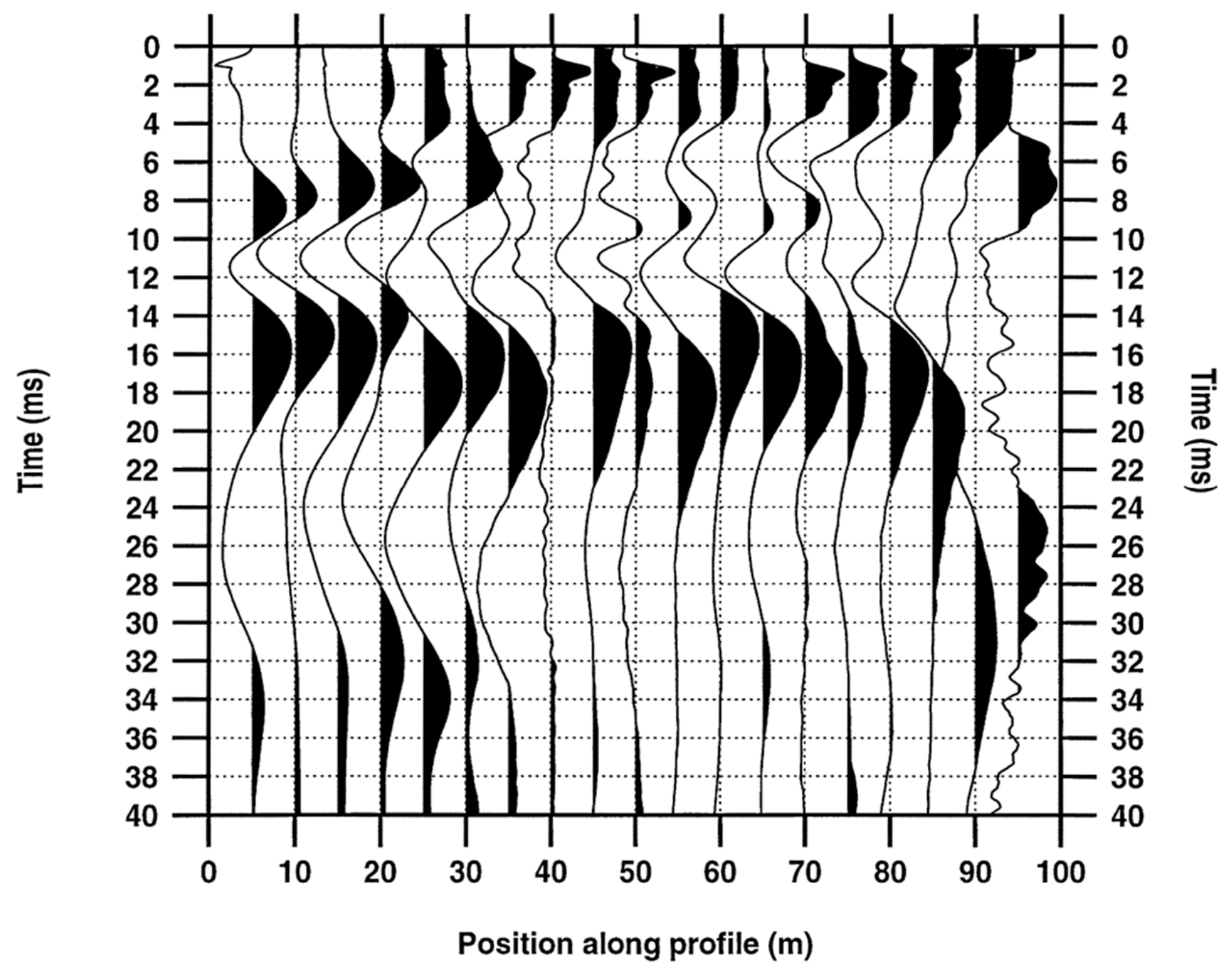

Figure 9. One-channel results (from a standard two-channel con-figuration) obtained at $5 \mathrm{~m}$ separations along a profile. Data are trace-normalized. 


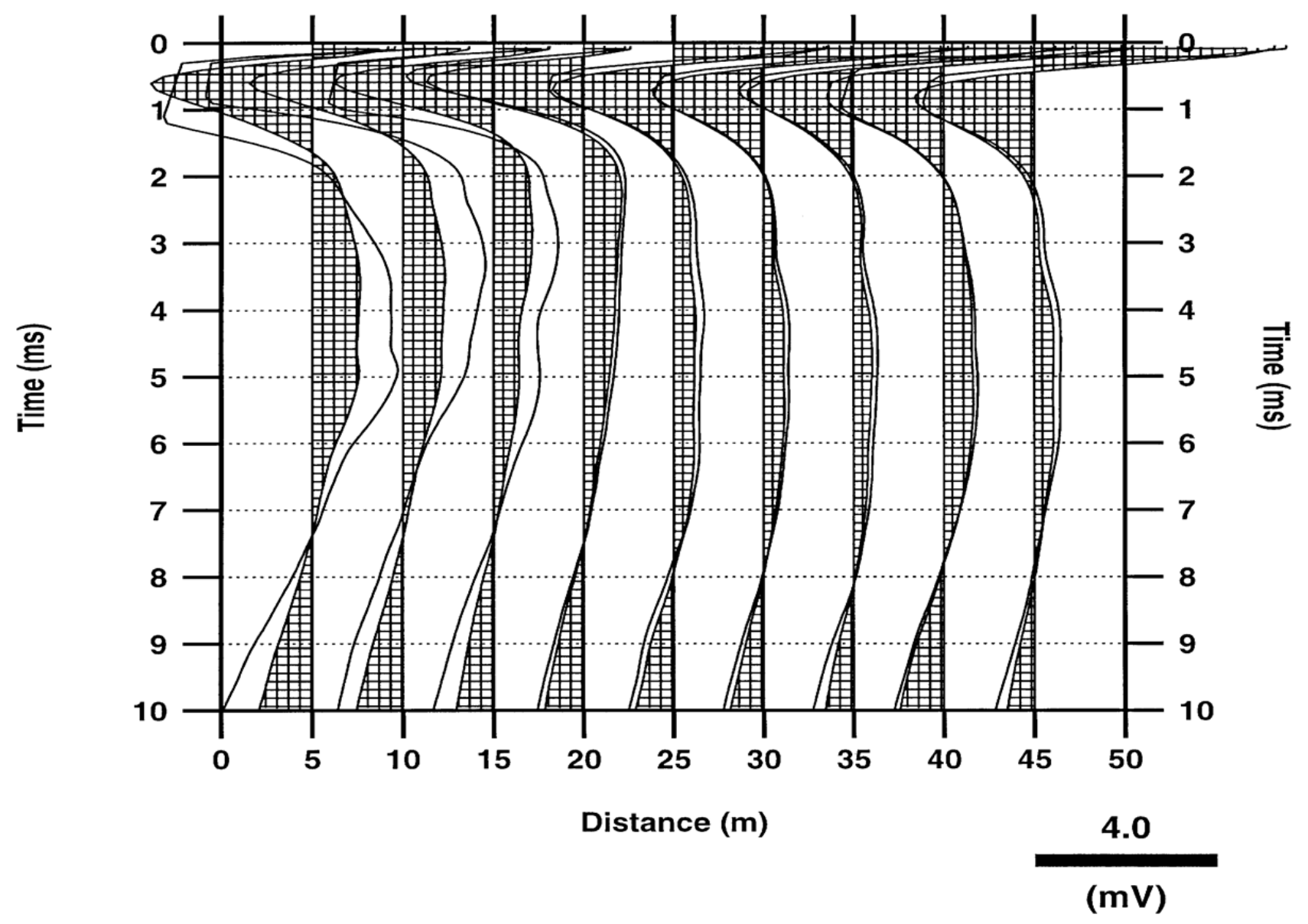

Figure 10. Two-channel results (from standard two-channel configuration) obtained at $5 \mathrm{~m}$ separations along a profile. Symmetric channels are jointly displayed as a single line trace and an infilled trace. Data are true amplitudes. 


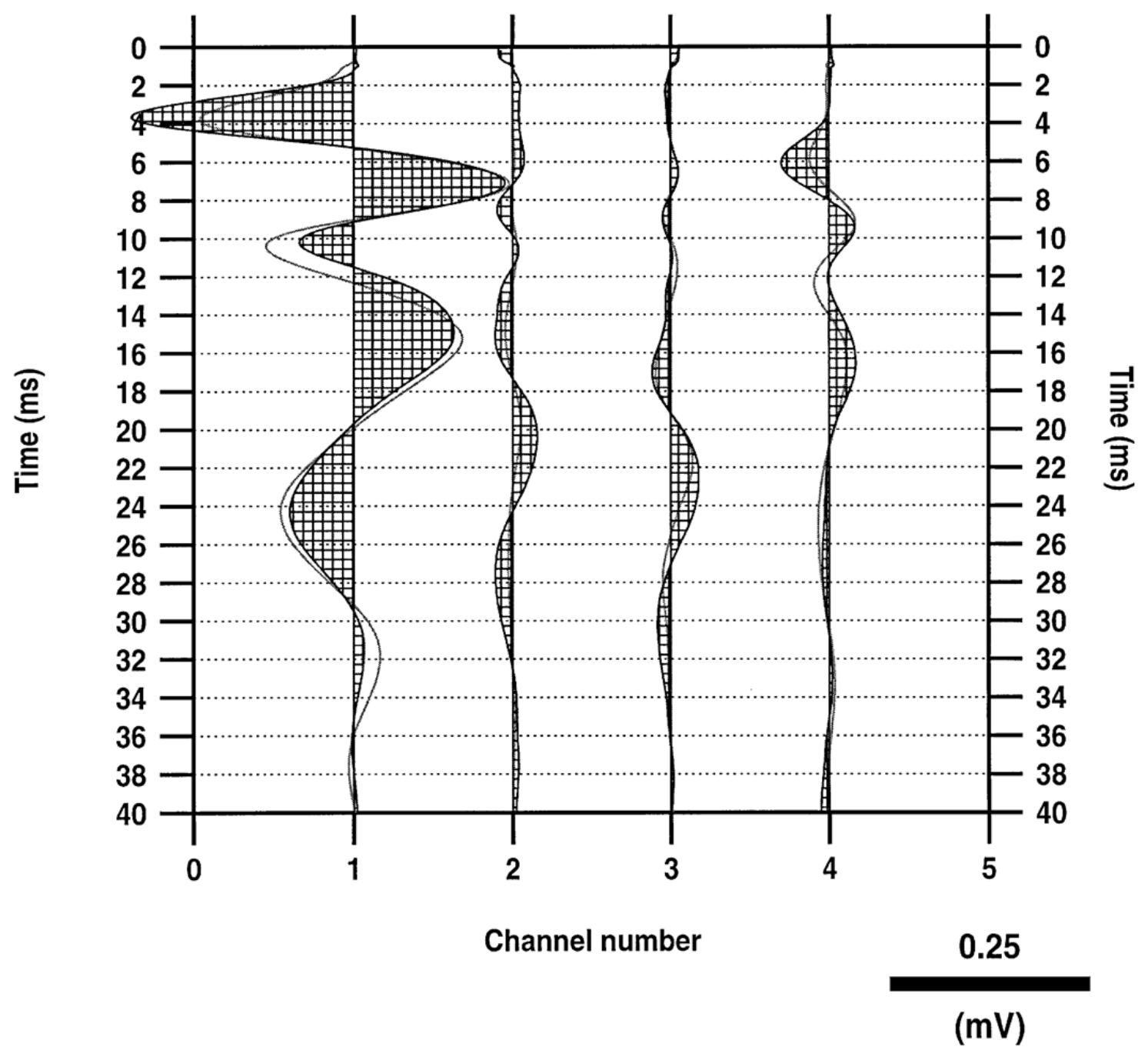

Figure 11. Example of an eight-channel recording displaying rapid spatial fall-o in amplitude. $2 \mathrm{~m}$ dipole centres are located at $\pm 1.5 \mathrm{~m}$ ( labelled 1), $\pm 2.5 \mathrm{~m}$ ( labelled 2), $\pm 3 \mathrm{~m}$ (labelled 3) from the shot- point. A 4$\mathrm{m}$ dipole centre is located at $\pm 3 \mathrm{~m}$ and is labelled 4 . Symmetric channels are jointly displayed as a single line trace and an infilled trace. 

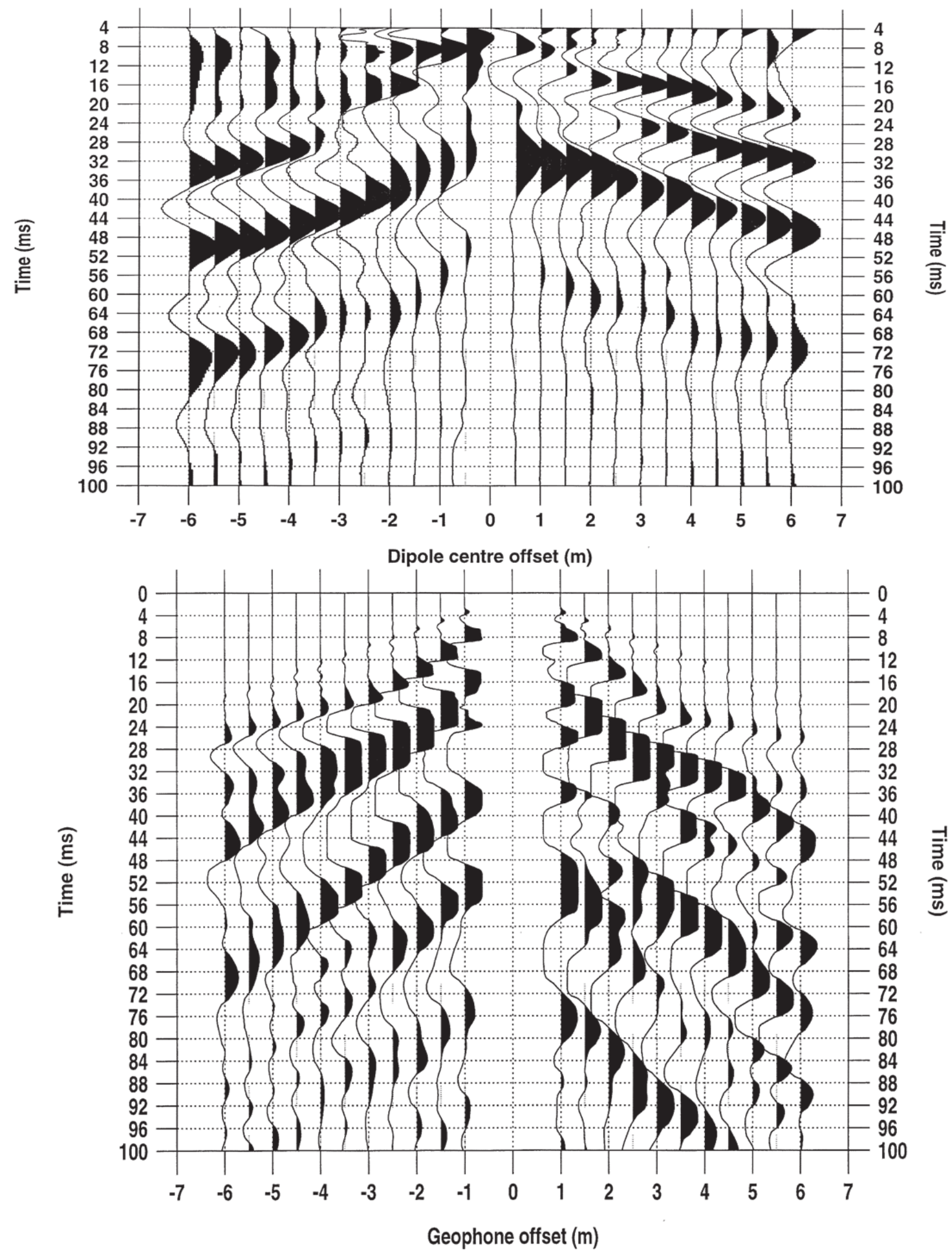

Figure 12. Example of joint electrokinetic/acoustic time-distance behaviour in the immediate vicinity of the shot-point. Boulder Clay above sandstone. Both data sets are shown as normalized traces. (a) Voltage data obtained using $0.5 \mathrm{~m}$ dipoles. ( b) Acoustic data obtained using $10 \mathrm{~Hz}$ vertical geophones. 


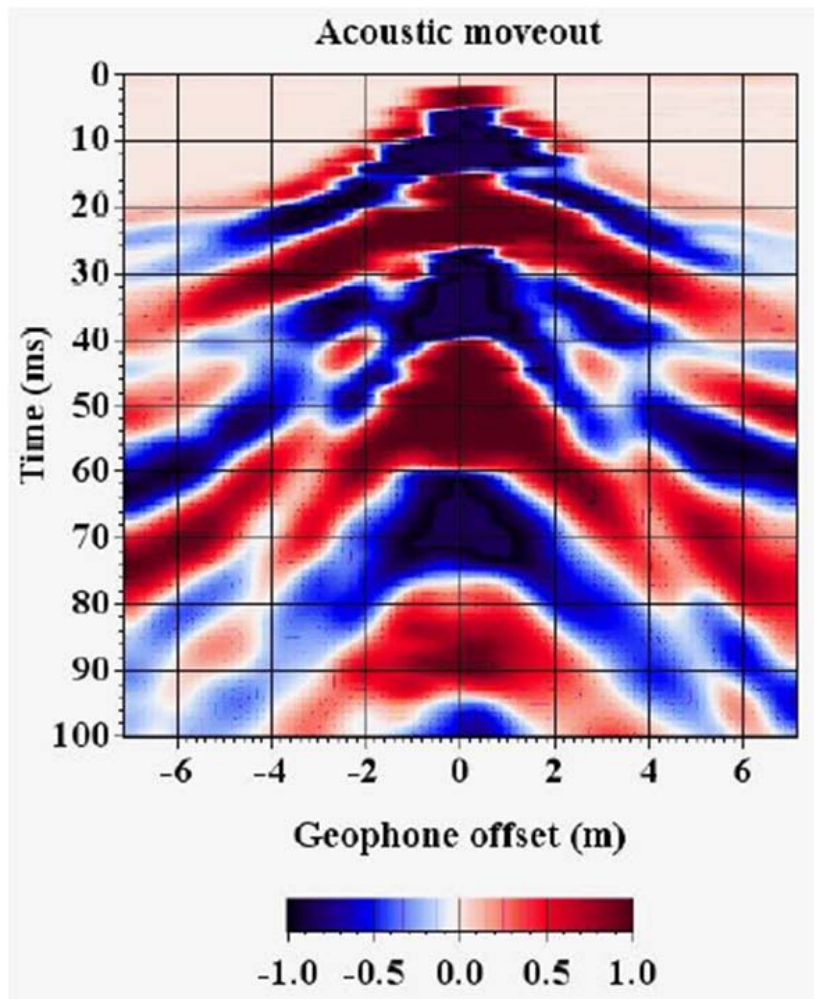

Normalised amplitude $(+/-)$
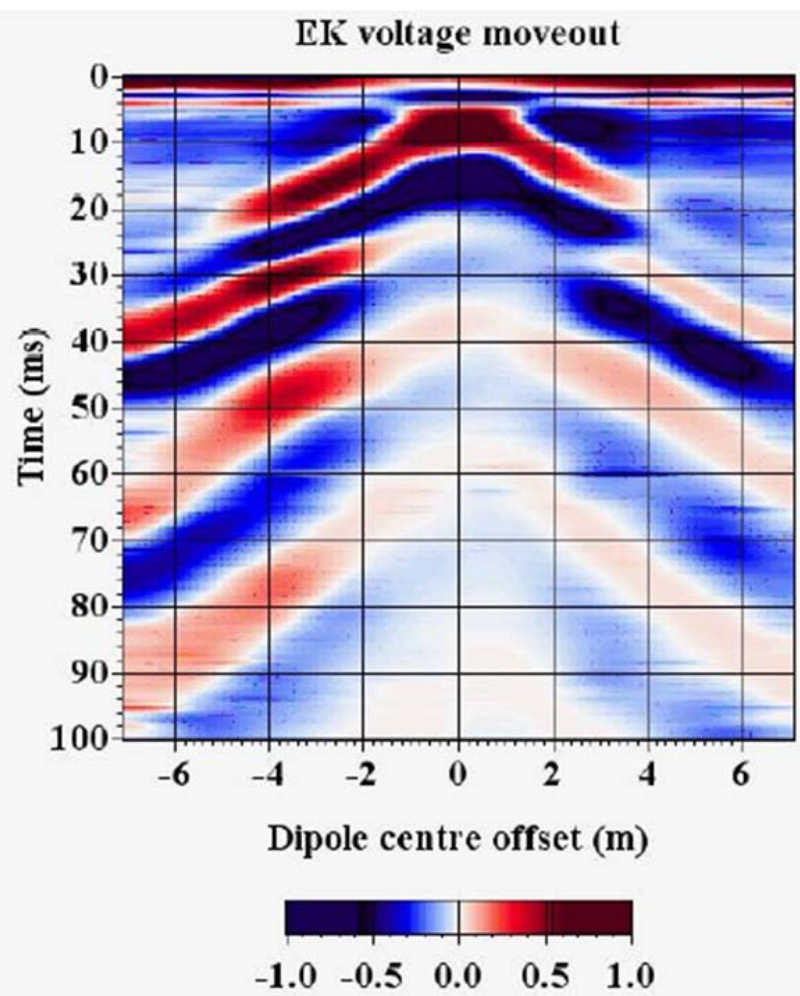

Normalised amplitude ( $+/$-)

Figure 13. Example of joint electrokinetic/acoustic time-distance behaviour in the immediate vicinity of the shot-point. Gore Sand River. Both data sets are shown as contoured normalized traces and were obtained at 0.5 m intervals. (a) Acoustic data obtained using $10 \mathrm{~Hz}$ vertical geophones. ( b) Voltage data obtained using $1.0 \mathrm{~m}$ dipoles. 
